\title{
Cutaneous Symptoms and Rashes in Patients with HIV-Positive Infections (A review article)
}

\author{
Mohammad Ali Shakeri Hosseinabad' and Farzad Abdolhazadeh ${ }^{2 *}$ \\ 'Resident of Dermatology, Ahvaz Jundishapur University of Medical Sciences, Ahvaz, Iran \\ ${ }^{2}$ Young Researchers and Elite Club, Boukan Branch, Islamic Azad University, Boukan, Iran; \\ f.abdolahzadeh@gmail.com
}

\begin{abstract}
Identifying the cutaneous symptom associated with HIV positive disease and investigating the kind of rashes can be helpful in early diagnosis of the disease. The extensity of skin disorders in HIV infection is widespread. Examination of the skin of these patients can be a good indicator of the status of the immune system as well as the patient's psychological status and also in early diagnosis can make a worthy help. We carried out a literature search (From 1990 to 2016) related to this issue through Google Scholar, Pub-MED, Scopus, Science Direct, and Iran DOC, by using keywords cutaneous symptoms, HIVpositive, skin infection, warts, early diagnosis. Among 124 articles only 51 papers were related to skin infections. Review of the associated research, investigation the skin infections in HIV patients and early diagnosis made a great help in the treatment process, particularly in people with high-risk behaviors which leads to diagnosis the HIV in the early stages. The results of this study suggest the importance of cutaneous symptom in patients with HIV infection and early diagnosis to start the medical procedures.
\end{abstract}

Keywords: Cutaneous Lesions, Early Diagnosis, HIV, Infection

\section{Introduction}

The HIV-positive disease is one of the most common skin infection diseases in this era. It can be said that is also one of the effects of human perversion of the moral regarding its common transmission way. Regarding the special facilities and special attention. In Western societies, now the extent of the epidemic has reached the constant level. In developing countries, especially in Asia, including our country regarding the increasing prevalence of the disease, more efforts to identify the symptoms, early diagnosis, control and prevention of disease is required ${ }^{1}$.

Since cutaneous symptoms are the indicators of HIV infection and the presence of these symptoms may be the first sign of HIV infection ${ }^{1}$ identifying the cutaneous symptom can cause the early diagnosis of the disease in the suspected cases.

\section{AIDS-Related Skin Infection Diseases and its Symptoms}

\subsection{Kaposi's Sarcoma}

Kaposi's sarcoma was considered as first cancer associated with AIDS. The disease, at first, appears in the form of pink macule and later published in the form of palpable patches, and has three stages: patch, plaque, and nodule. Mucous membrane involvement is common in this disease, but visceral diseases are common (about 10\% of patients suffer during diagnosis) and in the absence of symptoms, check for fetal diseases does not require ${ }^{1}$.

Clinical progress of the disease in AIDS patients is more aggressive than in patients with sarcoma without immunosuppression problem. Antiretroviral therapy complicated the prediction of disease (AIDS) by Kaposi's sarcoma, but it is believed that Kaposi's sarcoma as the 
first reported case of AIDS, as well as increasing the number of CD4 cells improve the prognosis of the disease process; while people over 50 years old with Kaposi's sarcoma cannot predict the disease process well ${ }^{1,2}$.

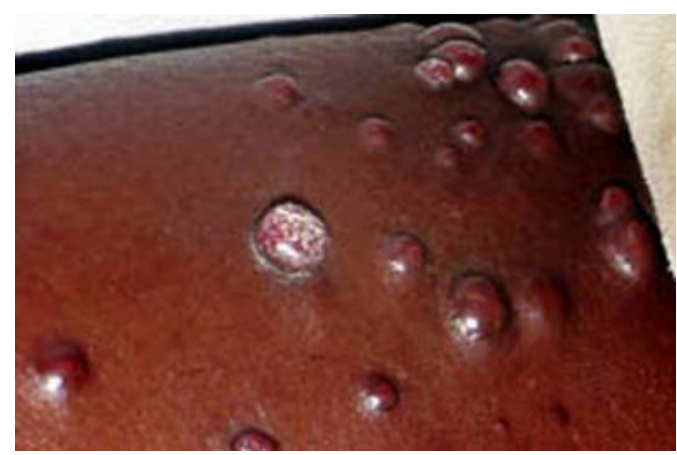

Figure 1. Kaposi sarcoma in a man with HIV infection. Adapted from Schwartz ${ }^{34}$.

\subsection{Other Malignancies}

AIDS increases the risk of various malignancies, such as, central nervous system (CNS) lymphoma, undifferentiated non-Hodgkin's lymphoma, squamous cell carcinoma, anal cancer and malignant skin tumors. With the increased use of antiretroviral therapy, the incidence of AIDS-defining cancers(Kaposi's sarcoma, non-Hodgkin's lymphoma and cervical cancer)which have a great relationship with immune suppression have been reduced. Of course, the total risk of cancer in these patients is still more than other people. The incidence of some of these cancers are associated with viruses such as Epstein-Barr virus and human papillomavirus ${ }^{1}$.

- B-cell non-Hodgkin lymphoma can cause skin nodules.

- AIDS causes anal carcinoma and cervical intraepithelial neoplasia (CIN).In this case they are more progressive and more aggressive.

- Sometimes in AIDS patients multiple squamous cell carcinoma of the oral cavity, Bowen's disease and basal cell carcinoma (BCC) is also seen. Basal cell carcinoma does not usually spread throughout the body. Of course, in AIDS patients the sudden onset of basal cell carcinoma have been reported, that after appearance of one lesion, several cases of basal cell carcinomas occur.

- Malignant melanoma is more aggressive in AIDS Patients.

- Children with HIV are more at risk ofleiomyosarcoma. Although this is rare but according to the research conducted in America, this disease has become the second most common malignant disease among children with AIDS or other immune deficiency diseases ${ }^{2}$.

\subsection{Viral Infections}

Different types of herpes simplex viruses are problematic for people with AIDS. They can occur herpetic lesions around the mouth and around the anus arising from herpes simplex virus, typical recurrent dermatomal zoster resulted from the herpes zoster virus and from disseminating infection of cytomegalovirus ${ }^{1,2}$.

- Oral and anogenital recurrent herpes simplex infection is common in AIDS patients and lead to chronic wounds. In children, swelling of the mouth caused by the herpes simplex virus is more common than varicella zoster virus and can cause chronic wounds.

- Varicella zoster virus infection diffusely happens with the unusual symptom. The symptoms are hyperkeratotic papules, folliculitis, wart lesions, ulcers, and released ecthymatouslesions and chronicvaricella zoster infections like basal cell carcinoma. Although varicella in children with AIDS makes little problems, but sometimes can cause sudden and severe chickenpox, pulmonitis, hepatitis and encephalitis.

- Epstein-Barr virus plays a role in oral leukoplakia. The virus causes a string of white papules on the sides of the tongue, that although it is not a precancerous factor, but it could be one of the signs of progressive suppression of the immune system. White patches with oral candidiasis may confuse with lichen planus and geographic tongue ${ }^{3}$.

- Cytomegalovirus causes ulcers in the perineum area. Through by the performance of the virus cannot predict the AIDS progress well.

- The widespread and resistant warts observed on the mucous lining of the mouth, face, perineum and genital area. The treatment of perineum area and cervical lesions is difficult (HPV-66 Human papillomavirus) and causes big warts on the soles of the feet.

- Molluscum contagiosum virus causes small papules with a small indentation in the center. In AIDS, the lesions caused by the virus are isolated and unusual. These lesions appear in unusual areas, such as the face, neck, and top of the head with unusual sizes and 
shapes, such as giant molluscum, endophyte, isolated, inflamed and masses ${ }^{3}$.

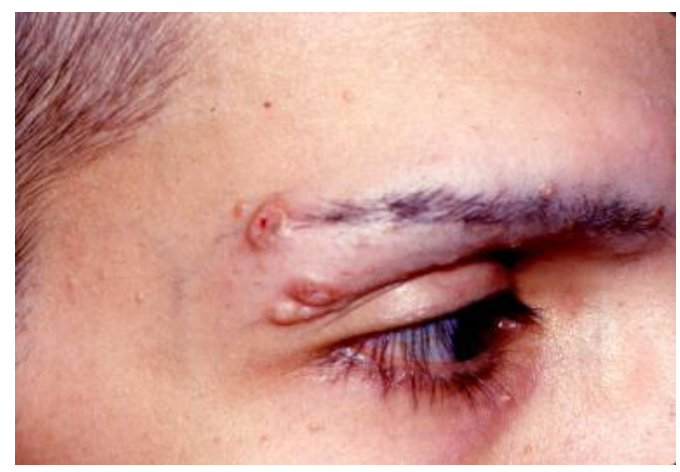

Figure 2. Young man with HIV disease and molluscum contagiosum on the lateral part of the eyebrow. Adapted from Schwartz ${ }^{34}$.

\subsection{Fungal Infections}

These infections can be superficial or deep.

- Resistant and recurrent candidiasis is common among AIDS patients. In America, vaginal candidiasis is the most common complication of AIDS infected women.

- In adults, extensive dermatophytosis, ortineacapitis, commonly caused by Trichophytonrubrum, can be a sign of HIV infection.

- Pityriasisversicolor can also occur in the form of persistent and recurrent.

- Deep fungal infections such as Coccidioidomycosis can also cause the skin lesions ${ }^{3,4}$.

\subsection{Bacterial Infections}

- Impetigo and folliculitis, especially in children, occur persistently and recurrently.

- It is possible that gingivitis, gangrenous stomatitis, abscesses and released boils occur.

- Tuberculosis(TB) is more common in people with AIDS. In many of these patients, Miliary Tuberculosis occurs that affects the skin. Mycobacterium infection is not only the tuberculosis factor but, in their native areas, can cause leprosy, sometimes occur in the form of lepromatous leprosy, because leprosy-associated tuberculosis causes the body's immune response.

- Bacillary angiomatos is caused by Bartonellahenselae and in rare cases by the Bartonellaquintana that usually produces red nodules and papules.

- Infections caused by unusual mycobacteria such as Mycobacterium avium.
- Syphilis had not been destroyed and yet appears in gay or bisexual or most of the consumers of illegal drugs. Many people with syphilis also are infected with HIV; this could be due to moral licentiousness and patients' lifestyle. Chronic wounds of syphilis make the HIV transmission easier. Usually, patients with primary syphilis and HIV compared with patients who do not have AIDS have more chronic wounds. AIDS causes the rapid progress into secondary syphilis and turn into tertiary syphilis. The serocon version for syphilis delayed and a standard serological test will not be reliable ${ }^{5}$.

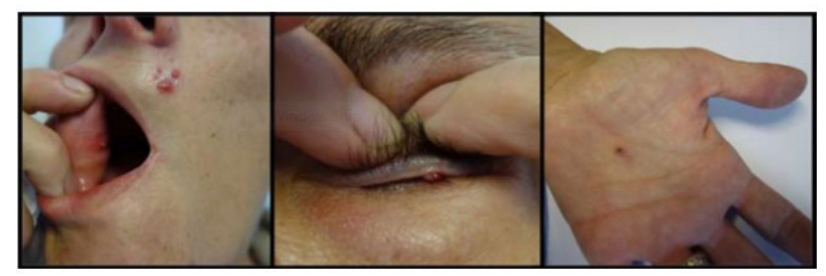

Figure 3. Bacillary angiomatosis, clinical presentation: Erythematous papules on the oral and left tarsal mucosa and on the palm of the left hand. Adapted from Lopes et al. ${ }^{35}$.

\subsection{Parasitic Infection}

The distinctive features of unusual or Norwegian scabies include extensive hyperkeratotic, scaly maculopapular rash or scaly plaques. Although this condition usually occurs in alcoholic people who do not follow health and likely have a problem in immune system, but also seen in AIDS patients ${ }^{6}$.

\subsection{Other Cutaneous Conditions}

- Many people with AIDS are infected with seborrheic dermatitis and similar complication. The condition can also be one of the symptoms of AIDS. This complication occurs with inflammatory lesions and widespread hyperkeratotic and in some patients turn to erythroderma. This complication occurs in patients with central nervous system disease or AIDS-related dementia.

- Psoriasis and arthritis are the more common reaction. In some cases, psoriasis intensifies and produces pustules and disseminated plaques, ${ }^{5,6}$.

- Skin lesions of Pityriasisrosea appear.

- Acquired Ichthyosis occurs in the legs and spread in advanced stages of the disease.

- Urticarial papules are one of the most common 
cutaneous rashes of AIDS. These small and itchy papules arered or skin-colored which appear on the face, neck and upper part of the trunk. Although, this complication is a strong sign of an advanced stage of immunosuppression.

- Stomatitis wounds can be severe $e^{5}$

- Thrombocytopenic purpura, vitiligo, alopecia areata, siccasyndrome, pemphigoid and otherautoimmune bullous diseases are associated with AIDS and have been seen with HIV disease.

- Atopic diseases will be reactivated. Atopic eczema can be severe in children.

- Urticariacan develop either primary or as a drugrelated skin disorder. Cold urticaria is also associated with AIDS.

- The incidence of cutaneous vasculitishas been reported.

- Sensitivity to light is more intense. Lichenoid drug reactions caused by sun exposure, andare observed,especially on dark skin.

\subsection{Hair and Nails}

- Diffuse alopecia or alopecia areata can occur permanently and inflammatory.

- Basic baldness occurs after the treatment with Indinavir, which is an antiretroviral agent.

- Lashes become long and hair becomes soft and smooth.

- Beau lines, telogen effluvium and yellow nail bed can occur with any chronic illness.

- Zidovudine can cause the longitudinal, transverse, melanin pigmentation of the nail. But the pigmentation of nails in those people with HIV who did not receive the drug has been observed.

- Early onychomycosis of beneath the nail symptoms is often associated with AIDS.

\subsection{Drug Reactions}

- About two-thirds of patients treated with cotrimoxazole are experiencingamorbilliform drug eruption. Today, doctors rarely prescribe this drug, but this is the best option for pneumonia caused by Pneumocystis jiroveciiwhich is an opportunistic infection commonly seen in AIDS patients. It's possible that after stopping treatment, reddish papules and macules become permanent ${ }^{7}$.

- It has been reported that in AIDS patients, antibiotics, fluconazole, clindamycin and phenobarbital cause the toxic epidermal necrolysis.

- Drug eruptions are the most common cause of erythroderma in patients with AIDS.

The most common cutaneous signs of HIV infection are caused by micro-organisms, among which the simple oral infections, herpes zoster, oral pityriasis candidate, molluscumcontagiosum and bacterial infection can be mentioned ${ }^{1}$.Oropharyngeal candidiasis is the most common fungal infection in patients with HIV infection and represents progressive disease ${ }^{1}$. According to a recent study ,herpes zoster infections in populations at risk of HIV, strongly suggests the possible implication of this virus ${ }^{2}$. AIDS for the first time was known as a new patient with Kaposi's sarcoma and Pneumocystis carinii pneumonia in homosexual men ${ }^{3}$. The existence of Human Immunodeficiency Virus in the blood is called (HIV) infection. Development of the disease which causes the cellular and humoral immunodeficiency is called AIDS. New criteria Center for Disease Control and Prevention (CDC) that contains a wealth of opportunistic infections which indicates severe cellular/humoral immune deficiency and also contains some cancers and other disorders are associated with severe immunodeficiency, the occurrence of any of these disorders in individuals that his immune deficiency has no other reason diagnosed as HIV infected. According to new CDC definitions, patient with severe immune deficiencies is an individual whose CD4 counts are less than 200 per cubic milliliter ${ }^{8}$.

Among the different symptoms of HIV disease, indications of cutaneous and mucosal are the most important clinical signs. Skin is one of the largest and most visible organs of the body that can usually show sign of internal diseases, in this issue about HIV infection is too. Although some of the skin lesions are non-specific, but with the progress of the disease, skin and mucous indications as clinical picture will be clearer as a result of the disease ${ }^{9}$.

In 1981,the first report of AIDS was reported, skin and mucosal diseases play a significant role in the clinical diagnosis of acquired immunodeficiency. HIV infection may be associated with various cutaneous symptoms that some of which are the important symptoms of the disease $^{10}$.

These symptoms may be infectious or non-infectious. Opportunistic infections that involved the skin can 
include viral, bacterial, fungal and protozoal agents. Noninfectious skin diseases are commonly associated with HIV infection and can intensify with foreign substances, such as drugs, or have an internal source, such as nonspecific pruritus ${ }^{11}$. Skin initial manifestation of AIDS is severe rash ${ }^{12}$.

Other indications such as urticaria and koplick's have also been reported ${ }^{13}$. Several studies also show folliculitis, abscesses, impetigo due to bacterial biological organisms that are caused by B-cells dysfunction ${ }^{14,15}$.

Fungal infections are very common in HIV infections. Oropharyngeal candidiasis (OPC) is one of the first symptoms of HIV infection ${ }^{16,17}$. Other uncommon fungal infections are also seen in these patients, such ascryptococcosis infections, valley fever, histoplasmosis and Aspergillus that act as systemic infections ${ }^{18,19}$.

In countries with easier access to Highly Active Antiretroviral Therapy [HAART], many physicians experience the spontaneous recovery of some of these infections such as OPC when the patients were treated with HARRT. But timely diagnosis and treatment of some of these fungal infections are critical to the patient ${ }^{20}$.

During recent two decades, AIDS has played a major role in adults and children's deaths. Many of these occur due to opportunistic infections or neoplasms. Kaposi's sarcoma and non-Hodgkin's lymphoma are two original mobile malignant diseases that associated with HIV infection. The epithelial cell cancers such as basal cell carcinoma (BCC) and squamous cell carcinoma (SCC) and malignant melanoma occur more in conjunction with HIV infection ${ }^{21}$.

To illustrate the importance of the issue, it is important to be mentioned that, these cancers are responsible against 2.2 million of African mortality ${ }^{18}$. Cutaneous symptoms of HIV are in close relationship with the number of $\mathrm{CD} 4^{+}$cells. For example, rashes occur when the number ofCD $4^{+}$between 500-1000, Tineacorporis, psoriasis, seborrheic dermatitis, impetigo in $\mathrm{CD}^{+}<500$, bacterial folliculitis, tineaversicolor, warts, molluscumcontagiosum and herpeszoster between 200 and $500 \mathrm{CD}^{+}$; herpes simplex, ichthyosis, follicular lesions, oral candidiasis, Kaposi's sarcoma and opportunistic infections occur at levels below $200 \mathrm{CD}^{+}$. This issue recognizes the importance of timely diagnosis of the disease ${ }^{22}$.

Although dermatologists play a major role in the diagnosis of infection, but many of the treatments are performed by general physicians, infectious disease specialists, and even oncologist. Most of these doctors specialize in the above have no ability to recognize the patient's skin indications especially various symptoms of disease which achieved only with a lot of work and experience. It has been recognized that sexually transmitted diseases, facilitate HIV transmission ${ }^{23}$.

The above mentioned issue states the importance of timely diagnosis of disease. In most patients, the diagnosis of an early stage of the disease is useful in estimating prognosis and for some individuals this issue plays a vital role $^{24,25}$.

Various studies conducted in different parts of the world, the incidence of cutaneous symptoms in these patients expressed very differently. In some, this figure reaches $90 \%{ }^{6}$. This type of symptoms and the prevalence are very different which it can be due to ethnic and geographical differences and different mode of transmission in different parts of the world ${ }^{26}$.

According to the World Health Organization, HIV infection in Iran is estimated to be about 70,000-100,000. Injection is the most important element of transmission of infection in Iran. About half of injecting drugs users got married and one-third of them have sexual relationships outside the family ${ }^{23}$. This shows the rapid spread of disease and stress the need for rapid diagnosis of disease detection. Two important external factors in the spread of HIV infection in Iran include

- Neighbors of Afghanistan, which is the largest producer of opium in Iran.

- In northern Iran situated some newly independent countries which have one of the fastest rates of HIV in the world ${ }^{27}$.

Due to the extraordinary importance of this disease, reviewed studies have been done on articles that examine the cutaneous symptoms associated with HIV. In fact, the skin is the largest organ in the body that is always exposed and its appearance reflects one's health. About 90 to 100 percent of HIV patients exhibit cutaneous signs and symptoms ${ }^{28,30}$.

The scope of skin disorders in HIV infection is widespread. Examination in these patients should be evaluated to assess immune function and mental status such as serological examination because the skin is also a good indicator of the immune system. The cause of skin lesions in these patients may be infectious (virus, bacteria, and fungi) and is non-infectious ${ }^{28}$. Common skin lesions 
such as warts, molluscumcontagiosum, herpetic lesions, tineaversicolor, impetigo and oral candidiasis worsen along the severity of immune function as well. Some skin conditions such as Kaposi's sarcoma are pathognomonic for this infection ${ }^{29,30}$. Evaluation and treatment of skin disorders in these patients are important in terms of improving the quality of life ${ }^{31}$. Since the determination of the prevalence of skin diseases and STDs in order to plan and deal with them is very important in these patients ${ }^{32,33}$. As well as detailed knowledge of the prevalence of skin diseases in HIV-positive individuals are not available, in the present review studies attempt to examine the signs of skin infection in HIV-positive patients.

\section{Findings}

According to the results of case studies is drawn from several specialized researches in this area estimated statistics of 70,000-1,00,000 people infected patients HIV/AIDS in Iran, it is expected that approximately 13500-9000 of Iranian patients with the disease have the associated skin manifestations ${ }^{28}$. Since the patient with HIV/AIDS is caused due to infection with HIV virus and the presence of Dermatoses with infectious causes, such as herpes zoster, warts and fungal infections and oropharyngealcandidiasis that a total of close to half of all Dermatoses observed in these patients are The value of close cooperation between the experts of the skin disease and infectious disease specialist in managing this patient is determined ${ }^{24}$.

With the arrival of the era of highly active antiretroviral therapy (HAART) and reduction of incidence of Dermatoses the mentioned colleagues receive more importance ${ }^{20}$. This management strategy, and it can be concluded that with respect to expose the skin and mucous membranes, and the association of HIV/AIDS with Skin and mucosal symptoms, it's required that group of healthcare providers such as doctors and dentists who are potentially at risk of early encounters with patients $^{33}$, have an appropriate information about these skin symptom so that if a patients with skin complaints referred to, particularly in patients with high-risk behaviors would not even stop to treat skin and does not lose HIV diagnosis in the early stages, because by HEART, it is possible to delay in entering the stage of AIDS ${ }^{8}$. The first review of the literature associated with the disease and skin infections in these patients by early diagnosis it will greatly assist in treatment, particularly in people with high-risk behaviors, and the HIV diagnosis in the early stages.

\section{Conclusion}

According to the results of review studies which examined on few articles in recent decades, it can be said that lesions and skin diseases are common in HIV-positive patients and most of these diseases are associated with the amount of $\mathrm{CD}^{+}$and the stage of the disease. Since some skin diseases are transmitted and in public health also are important, it's recommended that ${ }^{10}$, in HIV-positive cares system, the skin diseases should be included and patients have a regular skin examination ${ }^{25}$.

According to 33 case studies carried out in this regard can be said, most of the research on HIV-positive patients, which are under observation big clinics indicates that there is an urgent need for further research to be done with regard to the importance of the issue. In summary, this case study require attention to cutaneous symptoms in patients with HIV infection in Iran and early diagnosis of cutaneous manifestations in patients with HIV infection in Iran and early detection and screening, diagnosis and appropriate treatment were found.

\section{References}

1. Habif TP. Clinical dermatology. St Louis: Mosby; 1996. p.264-8.

2. Styer LM, Miller TT, Parker MM. Validation and clinical use of a sensitive HIV-2 viral load assay that uses a whole virus internal control. J ClinVirol. 2013; 58(Suppl 1):e12733.

3. Nasrullah M, Wesolowski LG, Meyer WA 3rd, et al. Performance of a fourth-generation HIV screening assay and an alternative HIV diagnostic testing algorithm. AIDS. 2013; 27(5):731-7.

4. Ananworanich J, Fletcher JL, Pinyakorn S, et al. A novel acute HIV infection staging system based on 4 th generation immunoassay. Retrovirology. 2013; 10:56.

5. Delaney KP, Branson BM, Uniyal A, et al. Evaluation of the performance characteristics of 6 rapid HIV antibody tests. Clin Infect Dis. 2011; 52(2):257-63.

6. Gulick R. Herpes virus infection. In: Arndt KA, LeBoit PE, Robinson JK, et al.,editors. Cutaneous medicine and surgery. Philadelphia: WB Saunders Company; 1996. p.107981.

7. Pneumocystis pneumonia--Los Angeles. Centers for Disease Control (CDC). MMWR Morb Mortal Wkly Rep. $1981 ; 30: 250-2$.

8. Zolpa AR, Katz MH. HIV Infection. In: Tierney LMJr, 
McPhee SJ, Papadakis MA,editors. Current Medical Diagnosis and Treatment. 46th ed. USA: McGraw-Hill Companies Inc; 2007. p. 1346-8.

9. Berger T. Cutaneous manifestations in patients infected with the human immunodeficiency virus. West J Med. 1996; 164: 516-7.

10. Kumarasamy N, Solomon S, Madhivanan P, et al. Dermatologic manifestations among HIV patients in south India. Int J Dermatol. 2000; 39:192-5.

11. Cockerell CJ, Freidman-kein AE. Cutaneous signs of HIV infection. In: Broder Sed. Text book of AIDS Medicine. Philadelphia, PA: Williams \&Wilkins; 1994. p. 507-24.

12. Tinndall B, Barker S, Donovan B, et al. Characterization of the acute clinical illness associated with HIV infection. Arch Intern Med. 1998; 148:945-6.

13. Kinlock S, de Saussure PIT, Vanhems PH, et al. Primary HIV infection: a prospective and retrospective study. Poster presented at: 8th International Conference on AIDS;1992 July 19-24; Amesterdam.

14. Siegal FP, Lopez C, Hammer GS, et al. Severe AIDS in male homosexuals, manifested by chronic perianal ulcerative herpes simplex lesions. N Eng J Med. 1981; 305:1439-44.

15. Berger TG, Sawchuk WS, Leonardi C, et al. Epidermodysplasiaverruciformis-associated papilloma virus infection complicating HIV disease. Br J Dermatol. 1991; 124:79-83.

16. Katzman M, Carey JT, Elmets CA, et al. MolluscumcontagiosumandAIDS.Clincal and immunological details of two cases. Br J Dermatol. 1987; 116:131-8.

17. Lin CS, Penha PD, Krishnan MN, Zak FG. Cytomegalic inclusion disease of skin. Arch Dermatol 1981; 117:282-4.

18. Wong D, Shumack S. Managing HIV. Part 5: Treating secondary outcomes. 5.1 HIV and skin disease. Med J Aust. 1996; 164:352-6.

19. DromerF, Dupont B.The increasing problem of fungal infections in the immune compromised host. J Mycol Med. 1996; 6:1-6.

20. Zingman BS. Resolution of refractory AIDS-related mucosal candidiasis after initiation of didanosine plus saquinavir. N Eng J Med. 1996; 334:1674-5.

21. Stebbing J, Gazzard B, Mandalia S, et al. Antiretroviral treatment regimens and immune parameters in the prevention of systemic AIDS-related NHL. J Clin Oncol. 2004; 22:2177-83.

22. USAID. Table of country-specific HIV /AIDS estimates and data, end 2003 July. [Accessed 4Sept 2009]. Available from: http://www.unaids.org/bangkok/.
23. Wong D, Shumack S. HIV and skin disease. In: Stewart G, editor. Managing HIV. North Sydney: Australasian Medical Publishing; 1997. p. 62-6.

24. Smith KJ, Skelton HG, Yeager J, et al. Cutaneous findings in HIV-1-positive patients: a 42-month prospective study. Military Medical Consortium for the Advancement of Retroviral Research (MMCARR). J Am Acad Dermatol. 1994; 31:746-57.

25. Tschachler E, Bergstresser PR, Stingl G, et al. HIV-related skin disease. Lancet. 1996; 348:659-3.

26. Freidman-Kien AE, Lafleur FL, Gendler E, et al. Herpes zoster: a possible early clinical sign for development of AIDS in high risk individuals. J Am Acad Dermatol. 1986; 14:1023-8.

27. USAID. Management's Discussion and Analysis 2006. Available from.

28. Supanaranond W, Desakorn V, Sitakalin C, Naing N, Chirachankul P. Cutaneous manifestations in HIVpositive patients. Southeast Asian J Trop Med Public Health. 2001 Mar; 32(1):171-6.

29. Schwartzman WA. Infections due to Rochalimaea: The expanding clinical spectrum. Clin Infect Dis. 1992; 15:893900.

30. Muhammad B, Eligius L, Mugusi F, Aris E, Chale S, Magao P, Josiah R, Moshi A, Swai A, Pallangyo N, Sandstrom E, Mhalu F, Biberfeld G, Pallangyo K. The prevalence and pattern of skin diseases in relation toCD4 counts among HIV-infected police officers in Dar es Salaam. Trop Doct. 2003 Jan; 33(1):44-8.

31. Spira R, Mignard M, Doutre MS, Morlat P, Dabis F. Prevalence of cutaneous disorders in a population of HIV-infected patients. Southwestern France, 1996. Grouped'Epidemiologie Clinique du SIDA en Aquitaine. Arch Dermatol. 1998 Oct; 134(10):1208-12.

32. Curtis SP, Edwards A. HIV in UK prisons: A review of seroprevalence, transmission and pattern of risk. IntJ STDAIDS. 1995; 6:387-91.

33. Murphy M, Gaffney K, Carey O, et al. The impact of HIV disease on Irish prison population. Int J STD-AIDS. 1992; 3:426-29.

34. Robert A Schwartz, Cutaneous Manifestations of HIV. 2016. Available from:http://emedicine.medscape.com/article/1133746-overview.

35. Leonor Lopes et al. Bacillary angiomatosis in a HIV-positive patient with poor adherence to antiretroviral therapy. Acta Dermatovenerol Croat.2014; 22(4):294-7. 